\title{
Estimation of Cardiovascular Risk from Self-Reported Knowledge of Risk Factors: Insights from the Minnesota Heart Survey
}

This article was published in the following Dove Press journal: Clinical Epidemiology

\author{
Sue Duval \\ Jeremy R Van't Hof' \\ Lyn M Steffen ${ }^{2}$ \\ Russell V Luepker ${ }^{2}$ \\ 'Cardiovascular Division, University of \\ Minnesota Medical School, Minneapolis, \\ MN, USA; ${ }^{2}$ Division of Epidemiology and \\ Community Health, School of Public \\ Health, University of Minnesota, \\ Minneapolis, MN, USA
}

Background: Cost-effective primary prevention of cardiovascular disease (CVD) relies on accuracy of risk assessment. Current risk scores require clinical and laboratory measures, are expensive and are often difficult to apply in the population setting.

Objective: This study sought to estimate CVD risk from individuals' knowledge of their own CVD risk factors and compare it to the risk calculated from measured risk factors.

Methods: Using the ACC/AHA Pooled Cohort Risk Equations (PCE), we calculated 10-year CVD risk for 9856 primary prevention individuals aged 40-79 in the Minnesota Heart Survey (MHS). Using log-linear regression models, we estimated PCE risk from the individual's selfreported knowledge of four dichotomous risk factors: hypertension, hypercholesterolemia, diabetes, and smoking. Age was included in all models, and models were developed separately in women and men. Model performance was assessed internally using leave-one-out cross-validation. Results: The median measured PCE CVD risk in women was 2.1\% (IQR: 0.8-5.6\%), and in men was $6.3 \%(3.1-13.0 \%)$. Using the newly developed equations, the median estimated risk was $2.2 \%(0.9-5.8 \%)$ in women, and $6.9 \%(3.2-13.1 \%)$ in men. Using a threshold of $7.5 \%$ to categorize low and high risk, the novel risk calculation gave an accuracy of $95 \%$ for women and $87 \%$ for men compared to the measured PCE risk. The negative predictive value was $97 \%$ for women and $91 \%$ in men.

Conclusion: Self-reported knowledge of risk may be useful in the identification of individuals at low risk for $\mathrm{CV}$ events, however, should always be followed up with measurement of risk factors if symptoms or history suggest CVD.

Keywords: cardiovascular diseases, epidemiology, risk assessment, risk factors, self-report, survey

\section{Introduction}

There are a growing number of self-assessment tools for prediction of risk for many different health conditions. They are designed to alert, educate, and change behavior, or direct people to health-care professionals. Historically these were paper and pencil tests given in clinics or community screenings, but they are now most often web-based, with some available on mobile phones. ${ }^{1}$ These self-assessments range from symptom analysis to calculation of disease risk.

Risk self-assessment surveys are widely used, as evidenced by the British tool for web-based cardiovascular risk calculation which was accessed 1.4 million times and taken by 575,782 users over 5 months in $2015 .^{2}$ However, many of these assessments, which often result in some type of "risk or health score", can be in
Correspondence: Sue Duval

Cardiovascular Division, University of Minnesota Medical School, 420 Delaware Street SE, MMC 508, Minneapolis, MN 55455 , USA

Tel +16126243392

Fax +16126264411

Emailsueduval@umn.edu 
error and misinterpreted. Self-misclassification can lead to inappropriate reassurance, whereas self-referral to health care for additional evaluation can lead to unnecessary expenses or treatment. Individuals may give answers they believe to be best, misinterpret the questions or not have actual data to enter.

Self-assessment of cardiovascular disease (CVD) risk is among the most common of these tools. ${ }^{3,4}$ Individuals without CVD can use these tools to predict their individual 10year risk of a cardiovascular event. The Framingham Risk Score had been the tool of choice for many years. ${ }^{4}$ The American Heart Association/American College of Cardiology Pooled Cohort Equations (PCE) risk calculator is also now widely available and designed for use in those with no history of CVD, and an LDL-C $<190 \mathrm{mg} / \mathrm{dl}^{3}{ }^{3}$ The PCE predict 10-year risk of coronary death, nonfatal myocardial infarction, fatal or nonfatal stroke. The PCE have been incorporated in national guidelines for CVD risk management for aspirin, hypertension and lipids.

The PCE risk calculator, however, will not calculate risk if any of the input values are missing. In a study by Van't Hof et al, ${ }^{5}$ only $43 \%$ of a primary prevention clinic population had complete data to allow PCE risk calculation using electronic health record data. The electronic health record data did contain ICD9 CM diagnostic codes for hypertension and hyperlipidemia, which, had they been incorporated, might have allowed many more individuals to be assessed. When risk assessment is not properly performed, high-risk individuals may be undertreated or low-risk individuals may inappropriately undergo a low-value screening test. As well, survey-based research assessing CVD risk becomes more expensive when laboratory and anthropometric data are required to complete the calculations.

Thus, this study was designed to evaluate a simpler format of CVD risk assessment using self-reported data on cardiovascular risk factors and demographics. We analyzed a random population sample of adults ages 40-79 years who answered questions about knowledge of their own cardiovascular risk factors, and who had blood pressure and cholesterol directly measured in a controlled research setting. In these analyses, we use regression methods to predict PCE risk using only participants' knowledge of risk.

\section{Methods}

\section{Sample}

The Minnesota Heart Survey (MHS) is a populationbased, serial cross-sectional study that assesses trends in cardiovascular disease risk factors in non-institutionalized resident adults aged 25 to 74 years in the Minneapolis/St. Paul (MSP) metropolitan area (2010 Census, 2.85 million). ${ }^{6}$ The MHS has completed six surveys (1980-1982, 1985-1987, 1990-1992, 1995-1997, 2000-2002, and 2007-2009). The MSP population is predominantly white and has slightly higher levels of education and employment than the United States population. MHS sampling was designed to obtain a random sample of the target population, using a two-stage cluster design created from census maps. The 7-county metropolitan area was divided into over 700 clusters of approximately 1000 households each. Forty clusters were randomly selected and used for each survey with a sample of households randomly selected from within each cluster. The census maps were updated before each survey to account for new housing developments and sampling was adjusted accordingly. In 1995, four clusters were added to represent new population growth and in 2007, three more clusters were added for a total of 47. Once a house was selected, it was removed from sampling in future surveys. After an introductory letter, selected households were visited by a trained interviewer who performed a household enumeration with those eligible selected for the survey. For this analysis, we used the combined MHS data from 1985 to 2009.

\section{Measurements in MHS}

Participants first took part in a home interview where interviewers collected data on demographic characteristics, cardiovascular medical history and risk factors, knowledge of cardiovascular risk factors, dietary habits, physical activity levels, health insurance coverage, access to a regular physician, self-rated health, smoking practices, and use of cardiovascular medications and dietary supplements. Participants were then invited to take part in a clinic visit. Data collected during the clinic visit included physiological measures such as anthropometric measurements, blood pressure, medical and reproductive history, and detailed questionnaires regarding diet, physical activity and smoking. Participants also underwent a non-fasting blood draw to assess serum total cholesterol and HDL cholesterol levels.

Each individual was asked four questions in their home to ascertain knowledge of risk: "Have you ever been told by a doctor or other health professional that you had high blood pressure?"; "Have you ever been told by a doctor or other health professional that you had high cholesterol?"; "Have you ever been told by a doctor or other health professional that you had diabetes?"; and for smoking, "Do you smoke at present?". Responses to these questions 
were either yes, no or do not know. We combined the negative responses, and any missing values, into a single category to represent no. Thus, possible numerical values for the individual risk factors were 0 and 1 , and their sum took on values of $0-4$, with 0 indicating that the participant reported having none of the four risk factors, to four where the participant responded as having all four risk factors.

The PCE 10-year CVD risk was calculated for each individual with complete data for each of the risk score components ( $\mathrm{n}=9856)$. Cholesterol was missing in $354(3 \%)$ participants and systolic BP in $316(3 \%)$. Individuals with total cholesterol $<130$ or $>320$; or HDL cholesterol $<20$ or $>100$; or $\mathrm{SBP}<90$ or $>200$; or $\mathrm{DBP}<30$ or $>140$ were excluded, because there are insufficient data to reliably predict risk in these cases. ${ }^{3}$

\section{Definition of Primary Prevention}

Individuals without a prior $\mathrm{CV}$ event were identified as those who responded "no" to the question "Have you ever been hospitalized for a heart attack or stroke?" Data for these individuals were collated from the 1985 to 2009 surveys and were restricted to those aged 40-79 years since that is one of the criteria for PCE assessment.

\section{Statistical Methods}

Multivariable linear regression models were developed to predict the measured PCE risk (dependent variable, mPCE) from individual's knowledge of risk (primary independent variable, knowledge of risk). To investigate the optimal parameterization for the four risk factor knowledge components, their sum was modeled as both a categorical variable (with five levels, 0-4) and as a continuous variable. The four risk factors were also modelled as separate dichotomous variables, taking the value of 0 (no) or 1 (yes). The mPCE risk was modeled on its native scale and was also log-transformed. Models were developed separately for men and women, and age was included as a linear and quadratic covariate. Adjusted $\mathrm{R}^{2}$ and Akaike's Information Criterion (AIC) were calculated from each model as measures of model fit, with better fitting models having a higher $\mathrm{R}^{2}$ and a lower AIC.

Estimated risk (ePCE) for each individual was calculated from the final log-linear models, using methods to avoid retransformation bias. ${ }^{7}$ Deciles of $\mathrm{mPCE}$ and $\mathrm{ePCE}$ were compared visually as a measure of calibration.

To assess characteristics of the ePCE, mPCE and ePCE were each divided into two categories: low risk $(<7.5 \%)$ and high risk ( $\geq 7.5 \%$ ), as was the risk threshold for statin therapy in the original work. ${ }^{3}$ Accuracy was calculated from each $2 \times 2$ table at a cutoff point of $\geq 7.5 \%$.

Internal validation was assessed by leave-one-out cross-validation, with measures of $\mathrm{R}^{2}$ and root-meansquared error (RMSE) averaged over each of the validation models.

Descriptive statistics for continuous variables are reported as mean \pm SD and categorical variables by frequency and percentage. All reported p-values are based on two-sided tests; a p-value of $<0.05$ was considered to indicate statistical significance. Analyses were performed in Stata Version 15.1 (StataCorp 2013. Stata Statistical Software Release 15. College Station, TX).

The Institutional Review Board of the University of Minnesota approved this study. The authors had full access to all of the data, and take responsibility for their integrity and the accuracy of the data analyses.

\section{Results}

Characteristics of individuals without a prior CV event, stratified by sex, are displayed in Table 1 . As there were limited numbers of individuals with a knowledge of risk sum of 4 (10 women, 8 men), those with 3 or 4 were combined. The MHS primary prevention population could be described as a low-risk CVD population, with close to $80 \%$ with 0 or 1 knowledge of risk sum, and the median $\mathrm{mPCE}<7.5 \%$.

Ten-year median (IQR) cardiovascular risk (\%) derived from the MPCE for each of the 16 possible combinations of the knowledge of risk factors is shown in Supplementary Table 1.

In Table 2, we show the strategy used to estimate PCE (ePCE) from only the individual's knowledge of their risk. The original PCE model (mPCE) includes race, but it was not used in this analysis, since the MHS population is $95 \%$ nonHispanic white. Both mPCE and ePCE contain the "same" risk factors, except that ePCE does not include whether the individual is taking an anti-hypertensive agent. The measured blood pressure and cholesterol in the $\mathrm{mPCE}$, requiring a clinic visit and a blood draw, are effectively "replaced" by knowledge of hypertension and hypercholesterolemia.

The risk profile as measured by the MPCE of MHS primary prevention individuals, stratified by age group and knowledge of risk sum is displayed in Figure 1 separately for women and men. Measured PCE increased with both age and knowledge of risk and is higher in men. 
Table I Characteristics of Minnesota Heart Survey Primary Prevention Population, 1985 to 2009. Age Range Restricted to 40-79 Years, and to Those Eligible for PCE Calculation ${ }^{a}$

\begin{tabular}{|c|c|c|c|}
\hline Characteristics & All & Women & Men \\
\hline $\mathrm{n}$ & 9856 & 5326 & 4530 \\
\hline Age & $53(10)$ & $54(10)$ & $53(10)$ \\
\hline Women & $5326(54 \%)$ & & \\
\hline \multicolumn{4}{|l|}{ Risk factors } \\
\hline Hypertension & $2844(29 \%)$ & 1496 (28\%) & $1348(30 \%)$ \\
\hline Hypercholesterolemia & $2850(29 \%)$ & 1403 (26\%) & 1447 (32\%) \\
\hline Diabetes & $496(5.1 \%)$ & $265(5.0 \%)$ & $231(5.1 \%)$ \\
\hline Current Smoking & $1695(17 \%)$ & $889(17 \%)$ & $806(18 \%)$ \\
\hline Measured systolic blood pressure & $123(16)$ & $|2|(17)$ & $125(15)$ \\
\hline Measured total cholesterol & $208(36)$ & $209(36)$ & $208(36)$ \\
\hline Measured HDL cholesterol & $49.5(14.8)$ & $55.4(14.5)$ & $42.6(11.8)$ \\
\hline Current anti-hypertensive & $1652(17 \%)$ & $903(17 \%)$ & $749(17 \%)$ \\
\hline Current anti-cholesterol agent & 749 (7.6\%) & $334(6.3 \%)$ & $415(9.2 \%)$ \\
\hline \multicolumn{4}{|l|}{ Number of risk factors ${ }^{b}$} \\
\hline 0 & $4 I 73(42 \%)$ & $2379(45 \%)$ & $1794(40 \%)$ \\
\hline I & $3797(39 \%)$ & 2005 (38\%) & $1792(40 \%)$ \\
\hline 2 & $1588(16 \%)$ & 788 (I5\%) & $800(18 \%)$ \\
\hline 3 or 4 & $298(3.0 \%)$ & $154(2.9 \%)$ & $144(3.2 \%)$ \\
\hline mPCE I0-year CV risk (\%) & $3.7(1.4,9.2)$ & $2.1(0.8,5.6)$ & $6.3(3.1,13.0$ \\
\hline
\end{tabular}

Notes: ${ }^{\mathrm{a}}$ Total cholesterol I30-320 mg/d; HDL 20-100 mg/dl; SBP 90-200 mmHg; DBP 30-140 mmHg. ${ }^{\mathrm{b}}$ Derived from knowledge of risk factors questions (see text). Values are mean (SD), $\mathrm{n}(\%)$, or median (25th, 75th percentile).

Abbreviations: HDL, high-density lipoproteins; mPCE, measured Pooled Cohort Equations I0-year CV risk.

Table 2 Description of mPCE and ePCE

\begin{tabular}{|l|l|l|}
\hline Risk "Score" & Model Input & Predicted Outcome \\
\hline Pooled cohort equations (mPCE) & $\begin{array}{l}\text { Age, smoking, DM, measured SBP, anti-hypertensive(s), } \\
\text { measured total cholesterol, HDL, race }\end{array}$ & $\begin{array}{l}\text { Coronary death or nonfatal myocardial } \\
\text { infarction, or fatal or nonfatal stroke }\end{array}$ \\
\hline Knowledge of risk (KoR) & $\begin{array}{l}\text { Age, smoking, knowledge of DM, hypertension, and } \\
\text { hypercholesterolemia }\end{array}$ & ePCE \\
\hline
\end{tabular}

Note: The original mPCE model includes race, but it was not used in this analysis (Minnesota Heart Survey is $95 \%$ Caucasian).

Abbreviations: DM, Diabetes; ePCE, estimated Pooled Cohort Equations I0-year CV risk; HDL, High-Density Lipoproteins; KoR, Knowledge of Risk factors; mPCE, measured Pooled Cohort Equations I0-year CV risk; SBP, Systolic BP.

Models using the log-transformed mPCE as the dependent variable performed uniformly better than the untransformed mPCE (results not shown). Supplementary Table 2 shows the adjusted $\mathrm{R}^{2}$ and Akaike's Information Criterion (AIC) for each of the models assessed. Models where knowledge of risk was included as four separate dichotomous variables were uniformly slightly better than the categorical or continuous (not shown) versions of the knowledge of risk.
The best models were chosen as those with the highest $\mathrm{R}^{2}$ and lowest AIC combination. The final models included, in women: high blood pressure, high cholesterol, diabetes mellitus, smoking, age (linear and quadratic terms) and the interaction of age with each of the dichotomous risk factors; in men, the model differed in that it only included the age interactions with high cholesterol and smoking, and not those with high blood pressure and diabetes mellitus. The same 


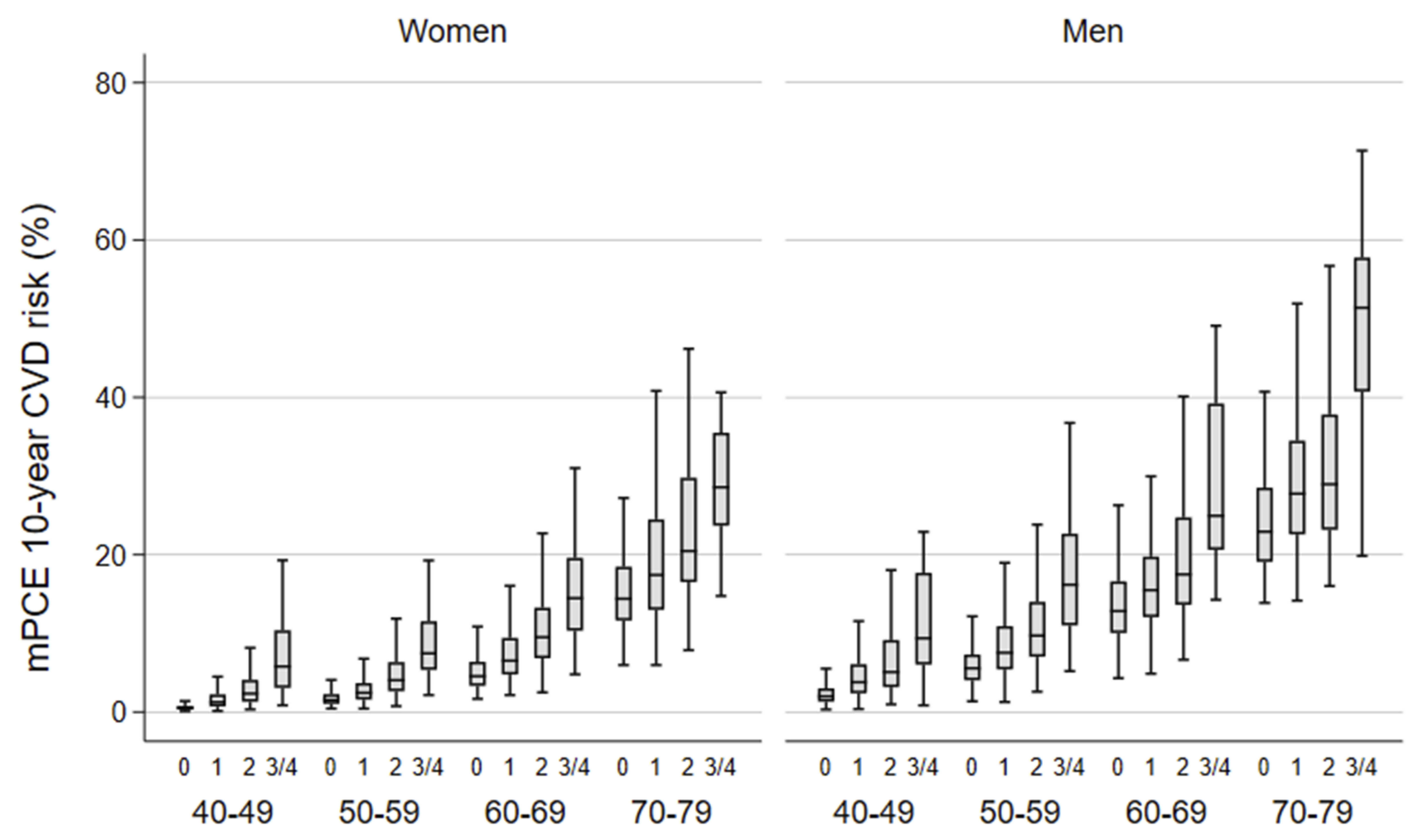

Figure I Measured PCE 10-year CVD risk (mPCE) by knowledge of risk and age group within women and men.

interaction models for women and men are shown for comparison purposes. Even though there is very little difference in the two interaction models in both women and men, the chosen models (highlighted in grey) performed better at higher levels of risk.

The derivation model regression coefficients for the candidate and final models are shown in Supplementary Table 3 and are given on the log-transformed scale for mPCE. The term for diabetes-age interaction was not statistically significant in women; however, its inclusion both decreased Akaike's Information Criterion (AIC) and improved model fit at higher levels of risk. Even though interpretation of the main effects is not straightforward in interaction models, it is clear that smoking in women, and smoking and age in men are powerful predictors of CVD risk.

Estimated risk (ePCE) for women can be calculated as $\exp (\Sigma \beta X-4.02)$, and for men as $\exp (\Sigma \beta X-5.8)$, where $\beta$ is the sex-specific regression coefficient and $\mathrm{X}$ is the level for each knowledge of risk factor (Supplementary Table 3). For example, for a 60 -year-old woman who currently smokes and has been told that she has high blood pressure, the ePCE risk is calculated to be $9.7 \%$.

The models showed good discrimination for the derivation model $\left(\mathrm{R}^{2}=0.87\right.$, root-mean-squared error $(\mathrm{RMSE})=0.46$ for women; $\mathrm{R}^{2}=0.82$, $\mathrm{RMSE}=0.41$ for men). Calibration of the models was assessed by scatterplots of mPCE and ePCE, superimposed with the four regions showing those who were concordant or discordant with regards to low $(<7.5 \%)$ or high $(\geq 7.5 \%)$ risk categories of each score (Figure 2$)$. There are relatively few discordant individuals $(5.5 \%$ in women and $12.6 \%$ in men). Thus, using the concordant cells to calculate accuracy gave values of $95 \%$ for women and $87 \%$ for men. Furthermore, the negative predictive value was $96.6 \%$ in women and $90.7 \%$ in men. When deciles of each of $\mathrm{mPCE}$ and $\mathrm{PCE}$ were calculated and plotted against each other, they showed remarkable correlation (Figure 3). The internal validation $\mathrm{R}^{2}$ and RMSE from leave-one-out cross validation were identical to the derivation statistics to two decimal places, again indicating good model fit.

Table 3 shows the measured and estimated PCE side by side, separately for women and men, and grouped by knowledge of risk sum and age group. There are some differences in the median values; however, they are relatively small and are more pronounced in older, higher risk individuals. In every case, the knowledge of risk model overestimates the median to some extent. These also correspond to cells with the smallest number of individuals and wider interquartile ranges. 

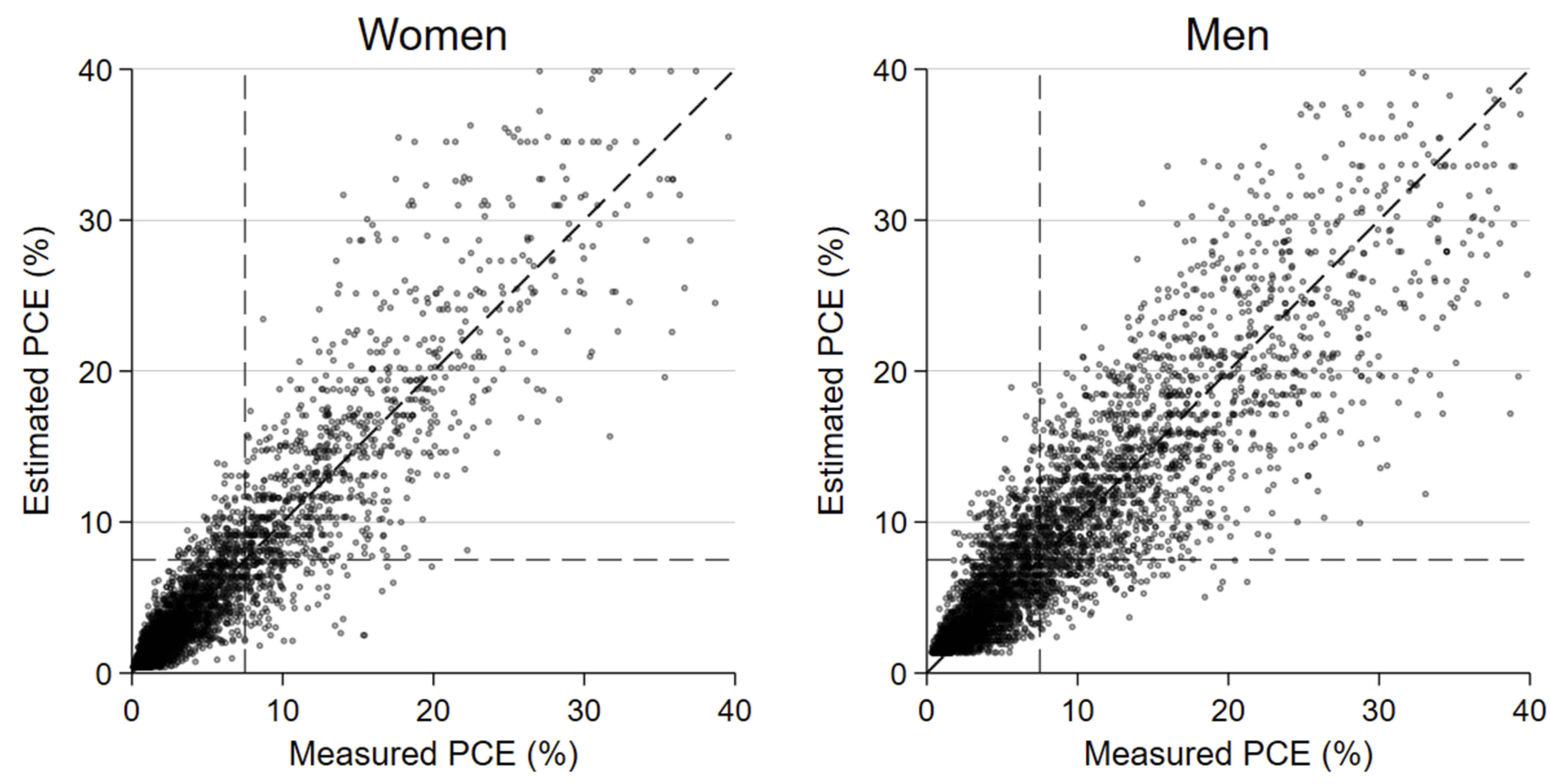

Figure 2 Estimated vs measured PCE 10-year CVD risk in women (left) and men (right). Values are only shown for those with mPCE<40.
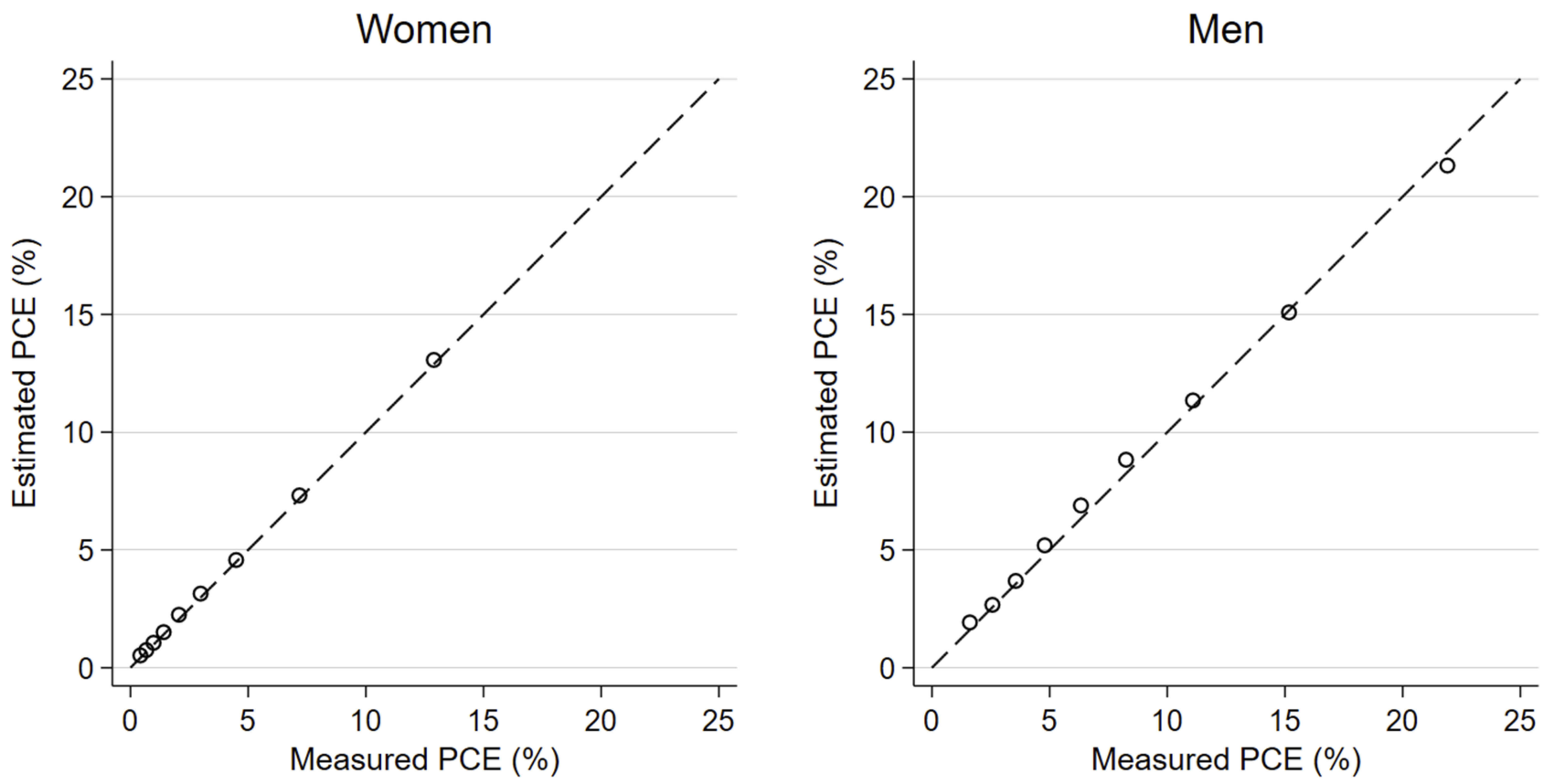

Figure 3 Calibration of the internal validation model, with line of identity denoting perfect calibration in women (left) and men (right). Risk is plotted at deciles of mPCE and ePCE.

\section{Discussion}

Using only simple individual knowledge of CVD risk, $95 \%$ of the women and $87 \%$ of the men were classified in concordance with their measured PCE risk. In the newly developed models, total and HDL-cholesterol measurements have been replaced by an individual's knowledge that a health-care professional told them they had high cholesterol. Systolic
BP and anti-hypertensive use in the PCE risk were replaced by whether the individual had been told they had hypertension. Smoking and diabetes variables are the same in measured PCE and models for estimation of PCE. Binary responses used as input to the newly developed knowledge of risk model were not derived from the measured blood pressure or cholesterol values in the MHS. 
Table 3 Measured (mPCE) and Estimated PCE (ePCE), Stratified by Age Group and Knowledge of Risk (Represented as the Sum of Risk Factors): Minnesota Heart Survey Primary Prevention Population, 1985 to 2009. Knowledge of Risk factors (KoR) are Hypertension, Hypercholesterolemia, Diabetes, and Smoking

\begin{tabular}{|c|c|c|c|c|c|}
\hline KoR & Risk & $40-49$ & $50-59$ & $60-69$ & $70-79$ \\
\hline \multicolumn{6}{|c|}{ Women } \\
\hline \multirow[t]{2}{*}{0} & $\mathrm{mPCE}$ & $0.5(0.4,0.8)$ & I.5 (I.0, 2.3) & $4.6(3.3,6.4)$ & $14.4(11.6,18.5)$ \\
\hline & ePCE & $0.6(0.5,0.8)$ & $1.6(1.3,2.3)$ & $5.0(3.9,6.4)$ & $17.0(13.2,20.6)$ \\
\hline \multirow[t]{2}{*}{1} & $\mathrm{mPCE}$ & I.3 $(0.7,2.2)$ & $2.4(1.6,3.7)$ & $6.5(4.8,9.4)$ & $17.4(13.0,24.5)$ \\
\hline & ePCE & I.3 $(0.9,2.5)$ & $2.7(2.0,3.8)$ & $7.4(5.2,9.2)$ & I8.8 (I4.7, 27.2) \\
\hline \multirow[t]{2}{*}{2} & mPCE & $2.3(1.3,4.1)$ & $4.0(2.7,6.3)$ & $9.5(6.8,13.3)$ & $20.5(16.5,30.0)$ \\
\hline & ePCE & $3.2(1.5,4.1)$ & $4.8(3.3,6.4)$ & $9.6(7.7,12.9)$ & $22.6(|6.9,3| .5)$ \\
\hline \multirow[t]{2}{*}{$3 / 4$} & mPCE & $5.8(3.1,10.4)$ & $7.5(5.4$, II.5) & $14.5(10.4,19.6)$ & $28.6(23.7,35.5)$ \\
\hline & ePCE & $6.2(4.9,7.8)$ & $8.6(7.8,10.1)$ & $14.9(12.3,19.9)$ & $36.5(27.4,45.5)$ \\
\hline \multicolumn{6}{|l|}{ Men } \\
\hline \multirow[t]{2}{*}{0} & mPCE & $2.0(1.3,3.0)$ & $5.6(4.1,7.3)$ & $12.8(10.1,16.6)$ & $22.9(19.1,28.5)$ \\
\hline & ePCE & $2.2(1.7,2.9)$ & $5.9(4.9,7.5)$ & I3.8 (II.I, I6.0) & $25.4(22.4,30.2)$ \\
\hline \multirow[t]{2}{*}{ I } & $\mathrm{mPCE}$ & $3.8(2.4,6.1)$ & $7.5(5.4,10.9)$ & $15.5(12.1,19.7)$ & $27.8(22.6,34.5)$ \\
\hline & ePCE & $3.8(2.6,6.0)$ & $8.4(6.1,10.5)$ & $16.8(13.5,18.9)$ & $28.7(26.2,33.6)$ \\
\hline \multirow[t]{2}{*}{2} & $\mathrm{mPCE}$ & $5.1(3.2,9.2)$ & $9.7(7.0,14.0)$ & $17.5(13.6,24.7)$ & $29.0(23.2,37.8)$ \\
\hline & ePCE & $5.7(3.6,8.7)$ & II.3 (8.3, 14.4) & $19.7(15.7,23.4)$ & $30.9(27.8,37.0)$ \\
\hline \multirow[t]{2}{*}{$3 / 4$} & $\mathrm{mPCE}$ & $9.4(6.0,17.7)$ & $16.2(11.0,22.7)$ & $24.9(20.6,39.2)$ & $51.4(40.7,57.8)$ \\
\hline & ePCE & $10.6(8.8,13.1)$ & $18.9(15.5,20.9)$ & $27.2(25.2,37.3)$ & $54.9(47.1,61.9)$ \\
\hline
\end{tabular}

Note: Values are median (25th, 75th percentile) of 10 -year CV risk (\%).

Abbreviations: ePCE, estimated Pooled Cohort Equations 10-year CV risk; KoR, knowledge of risk factors; mPCE, measured Pooled Cohort Equations I0-year CV risk.

Unlike most risk prediction models, the models derived here were without knowledge of cardiovascular events. Cardiovascular outcomes were not available in the MHS; however, data were available to simultaneously allow the computation of mPCE, as well as yes or no responses to questions about knowledge of risk. When faced with selfreport data where calculation of the $\mathrm{mPCE}$ is impossible, we have shown that these models have good statistical properties, and thus could be used to quantify risk when laboratory data are unavailable. Used as a screening assessment in asymptomatic adults, a low ePCE may be useful in reducing unnecessary testing. With very high negative predictive values of over $96 \%$ in women and $90 \%$ in men, the newly developed score can potentially identify low-risk individuals who may not require further evaluation (eg, laboratory testing, coronary artery calcium (CAC) score). This will require additional evaluation. Currently, any clinical decision to modify an individual's treatment plan due to CVD risk should be well informed by the appropriate blood pressure, laboratory and radiologic testing.
The four risk factors are not evenly weighted in their contribution to CVD risk, with some combinations uncommon in these data. This explains why the individual risk factors performed better than the sum of risk factors in estimation models.

In a nationwide survey of adults aged 45-75, "objective risk" of CVD was ascertained based on age, sex, and number of self-reported CVD risk factors. ${ }^{8}$ Four of the five risk factors used in this survey were the same as we used in this analysis, with the added self-report of family history of stroke, angina, heart attack or heart surgery. We believe that our method goes one step further than this work in attempting to model these factors to estimate absolute risk.

We do not know from this study how well the knowledge of risk model will predict CVD outcomes. Further study in an appropriate data set is needed to answer this question; however, perhaps it may perform better than the measured PCE. Measurements of blood pressure and cholesterol for input to the $\mathrm{mPCE}$ risk calculator are typically 
from a single measurement, whereas knowledge of these risk factors may be more reliable and stable.

\section{Limitations}

The MHS data used in these analyses were obtained from primary prevention individuals living in the Twin Cities metropolitan area from 1985 to 2009. During this period, risk factors for CVD have changed, with notable reductions in smoking from $23 \%$ to $11 \%$. Statin use in the MHS study increased from $1 \%$ in 1985 to $19 \%$ in 2009 , somewhat in alignment with the self-report of high cholesterol which increased from $15 \%$ to $41 \%$. Despite these ecologic trends, the predicted PCE was remarkably stable across survey years.

Measured PCE risk has been shown to overestimate CVD risk, ${ }^{9}$ and we have shown that knowledge of risk tends to overestimate the mPCE. However, when we classified high CVD risk based on a cutoff of 7.5\% risk, measured PCE predicted that 19\% women and 44\% men would be classified as high cardiovascular risk and the corresponding knowledge of risk estimates were very similar, $19 \%$ and $46 \%$, respectively. Until further evaluation, we would recommend that the knowledge of risk be best used for dividing individuals into risk categories, where different cut-points could be used depending on the research question, and tolerance of false negatives and false positives.

The majority of our sample had low CVD risk. Internal validation showed excellent calibration even in the highrisk deciles, but more work should be done to validate the knowledge of risk score in an intermediate to high risk sample.

\section{Statistical Limitations}

Statistically, the models are by definition multicollinear. The knowledge of risk as parameterized in the final models as four separate factors can only take the values of 0 and 1 , and their interactions with age can only take the values of 0 and age. With such a large data set, these effects are less likely to be problematic, and the models performed well in terms of estimated standard errors.

Performance of a predictive model is typically overestimated when simply determined on the sample of subjects that was used to construct the model. We showed that using leave-one-out cross-validation, this bias was very small. Still, recalibration of the knowledge of risk model in a different dataset may be necessary, such as was done for the Framingham prediction scores. ${ }^{10}$

This work does not include external validation of the newly developed knowledge of risk models to estimate mPCE. Nor does it use cardiovascular events in its development, such as those outcomes predicted by the mPCE risk. The next step in the development of these models for practical use is to externally validate them in other primary prevention populations.

\section{Conclusions}

The knowledge of risk score provides a highly accurate method of estimating CVD risk without the need for blood pressure or laboratory data. This method of calculating risk may be useful in research settings or electronic health record analyses where such data are not readily available.

\section{Disclosure}

The authors report no conflicts of interest in this work.

\section{References}

1. ASCVD risk estimator [Internet]. Available from: tools.acc.org/ ASCVD-Risk-Estimator-Plus. Accessed December 03, 2019.

2. Patel RS, Lagord C, Waterall J, Moth M, Knapton M, Deanfield JE. Online self-assessment of cardiovascular risk using the Joint British Societies (JBS3)-derived heart age tool: a descriptive study. $B M J$ Open. 2016;6:e011511. doi:10.1136/bmjopen-2016-011511

3. Goff DC, Lloyd-Jones DM, Bennett G, et al. 2013 ACC/AHA guideline on the assessment of cardiovascular risk: a report of the American College of Cardiology/American Heart Association task force on practice guidelines. Circulation. 2014;129:S49-S73. doi:10.1161/01.cir.0000437741.48606.98

4. D'Agostino RB, Vasan RS, Pencina MJ, et al. General cardiovascular risk profile for use in primary care. Circulation. 2008;117:743-753. doi:10.1161/CIRCULATIONAHA.107.699579

5. Van't Hof JR, Duval S, Walts A, Kopecky SL, Luepker RV, Hirsch AT. Contemporary primary prevention aspirin use by cardiovascular disease risk: impact of US preventive services task force recommendations, 2007-2015: a serial, cross-sectional study. $J \mathrm{Am}$ Heart Assoc. 2017;6:1-12.

6. Luepker RV, Steffen LM, Jacobs DR, Zhou X, Blackburn H. Trends in blood pressure and hypertension detection, treatment, and control 1980 to 2009: the Minnesota Heart Survey. Circulation. 2012;126 (15):1852-1857.

7. Beauchamp JJ, Olson JS. Corrections for bias in regression estimates after logarithmic transformation. Ecology. 1973;54:1403-1407. doi: $10.2307 / 1934208$

8. Williams CD, Chan AT, Elman MR, et al. Aspirin use among adults in the U.S.: results of a national survey. Am J Prev Med. 2015;48:501-508. doi:10.1016/j.amepre.2014.11.005

9. Ridker PM, Cook NR. Statins: new American guidelines for prevention of cardiovascular disease. Lancet. 2013;382:1762-1765.

10. D'Agostino RB Sr, Grundy S, Sullivan LM, Wilson P. Validation of the Framingham Coronary Heart Disease prediction score. $\mathrm{J} \mathrm{Am} \mathrm{Med}$ Assoc. 2001;286:180-187. doi:10.1001/jama.286.2.180 


\section{Publish your work in this journal}

Clinical Epidemiology is an international, peer-reviewed, open access, online journal focusing on disease and drug epidemiology, identification of risk factors and screening procedures to develop optimal preventative initiatives and programs. Specific topics include: diagnosis, prognosis, treatment, screening, prevention, risk factor modification,

Submit your manuscript here: https://www.dovepress.com/clinical-epidemiology-journal systematic reviews, risk \& safety of medical interventions, epidemiology \& biostatistical methods, and evaluation of guidelines, translational medicine, health policies \& economic evaluations. The manuscript management system is completely online and includes a very quick and fair peer-review system, which is all easy to use. 